\title{
UNIQUENESS OF $L^{1}$ SOLUTIONS FOR THE LAPLACE EQUATION AND THE HEAT EQUATION ON RIEMANNIAN MANIFOLDS
}

\author{
PETER LI
}

In this paper, our goal is to derive an optimal geometrical assumption on a complete Riemannian manifold which will ensure uniqueness properties for $L^{1}$ solutions of the Laplace equation and the heat equation. We say that a manifold has Ricci curvature with negative quadratic lower bound if there exists a point $x_{0} \in M$ and a constant $C>0$, such that the Ricci curvature at any point $x \in M$ satisfies

$$
\operatorname{Ric}(x) \geqslant-C\left(1+r^{2}(x)\right)
$$

where $r(x)$ denotes distance from $x_{0}$ to $x$. It turns out that the above Ricci curvature condition is the optimal assumption to guarantee uniqueness in both equations. In fact, we prove the following theorems.

Theorem 1. Let $M$ be a complete noncompact Riemannian manifold without boundary. If the Ricci curvature of $M$ has a negative quadratic lower bound (1), then any $L^{1}$ nonnegative subharmonic function on $M$ must be identically constant. In particular, any $L^{1}$ harmonic function on $M$ must be identically constant.

Theorem 2. Let $M$ be a complete noncompact Riemannian manifold without boundary. If the Ricci curvature of $M$ has a negative quadratic lower bound (1), and if $v(x, t)$ is a nonnegative function defined on $M \times[0, \infty)$ satisfying

$$
\left(\Delta-\frac{\partial}{\partial t}\right) v(x, t) \geqslant 0, \quad \int_{M} v(x, t) d x<\infty
$$

for all $t>0$, and

$$
\lim _{t \rightarrow 0} \int_{M} v(x, t) d x=0,
$$

then $v(x, t)=0$ for all $x \in M$ and $t \in(0, \infty)$.

Received June 11, 1984. This research was partially supported by a Sloan fellowship and a National Science Foundation grant. 
In particular, any $L^{1}$ solution of the heat equation on $M$ is uniquely determined by its initial data in $L^{1}(M)$.

We would like to point out that in [6], the author and Schoen proved that if the Ricci curvature of $M$ satisfies

$$
\operatorname{Ric}(x) \geqslant-C\left(1+r^{2}(x)\right)\left[\log \left(1+r^{2}(x)\right)\right]^{-\alpha},
$$

then $M$ does not admit any nonconstant nonnegative $L^{1}$ subharmonic function. In the same article, an example was given with sectional curvature behaving like

$$
K(x) \sim-C r^{2+\varepsilon}(x)
$$

for $C, \varepsilon>0$, and admits nonconstant nonnegative $L^{1}$ harmonic functions. Hence Theorem 1 can be viewed as a sharp improvement of the theorem in [6]. Uniqueness for $L^{p}$ nonnegative subharmonic functions were proved in [10] and [6] for the cases $p \in(1, \infty)$ and $p \in(0,1)$ respectively. Nonexistence of bounded harmonic functions was proved by Yau in [9], and the case of bounded subharmonic functions was discussed in [7]. We refer to [6] for a more detailed account on the history of the subject.

As for the case of the heat equation, uniqueness of bounded solutions and nonnegative solutions were proved in [5] and [4], [7] respectively. When $p \in(1, \infty)$, similar to the case of nonnegative subharmonic function, uniqueness is automatic for any complete manifold as indicated by the following

Proposition. Let $M$ be a complete Riemannian manifold. If $v(x, t)$ is a nonnegative function defined on $M \times[0, \infty)$ with

$$
\left(\Delta-\frac{\partial}{\partial t}\right) v(x, t) \geqslant 0, \quad \int_{M} v^{p}(x, t) d x<\infty
$$

for all $t>0$, and

$$
\lim _{t \rightarrow 0} \int_{M} v^{p}(x, t) d x=0,
$$

then $v(x, t)=0$ for all $x \in M$ and $t>0$.

In particular, any $L^{p}$ solution of the heat equation is uniquely determined by its initial data in $L^{p}(M)$.

Proof. Let $\varphi(x)$ be a cut-off function with the property that

$$
\varphi(r(x))= \begin{cases}1 & \text { on } B_{x_{0}}(R), \\ 0 & \text { off } B_{x_{0}}(2 R),\end{cases}
$$


with $0 \leqslant \varphi \leqslant 1$ and $|\nabla \varphi| \leqslant 3 / R$, where $r(x)$ denotes distance from $x$ to $x_{0}$ and $B_{x_{0}}(R)$ is the geodesic centered at $x_{0}$ with radius $R$. Consider

$$
\begin{aligned}
\int_{0}^{T} \int_{M} \varphi^{2}(x) v^{p-1}(x, t) \Delta v(x, t) d x d t \\
\quad \geqslant \int_{0}^{T} \int_{M} \varphi^{2} v^{p-1} \frac{\partial v}{\partial t} d x d t \\
\quad=\frac{1}{p} \int_{0}^{T} \frac{\partial}{\partial t}\left(\int_{M} \varphi^{2} v^{p} d x\right) d t=\frac{1}{p} \int_{M} \varphi^{2}(x) v^{p}(x, T) d x,
\end{aligned}
$$

by the assumption on $v$. On the other hand, integrating by parts gives

$$
\begin{aligned}
& \int_{0}^{T} \int_{M} \varphi^{2} v^{p-1} \Delta v \\
& \quad=-2 \int_{0}^{T} \int_{M} \varphi v^{p-1}\langle\nabla \varphi, \nabla v\rangle-(p-1) \int_{0}^{T} \int_{M} \varphi^{2} v^{p-2}|\nabla v|^{2} \\
& \quad \leqslant \frac{2}{(p-1)} \int_{0}^{T} \int_{M}|\nabla \varphi|^{2} v^{p}-\frac{2(p-1)}{p^{2}} \int_{0}^{T} \int_{M} \varphi^{2}\left|\nabla\left(v^{p / 2}\right)\right|^{2}
\end{aligned}
$$

Hence combining with (2) and the assumption on $|\nabla \varphi|$, we have

$$
\int_{M} \varphi^{2} v^{p}+\frac{2(p-1)}{p} \int_{0}^{T} \int_{M} \varphi^{2}\left|\nabla\left(v^{p / 2}\right)\right|^{2} \leqslant \frac{6 p}{(p-1) R^{2}} \int_{0}^{T} \int_{M} v^{p} d x .
$$

Letting $R \rightarrow \infty$, we obtain both

$$
\int_{M} v^{p}(x, T) d x=0 \text { and } \int_{0}^{T} \int_{M}\left|\nabla\left(v^{p / 2}\right)\right|^{2}=0
$$

hence $v \equiv 0$.

We would like to point out that when $p \in(1, \infty)$, the uniqueness of strongly continuous contractive $L^{p}$ semigroup for the heat equation was proved by Strichartz in [8]. In [6], the uniqueness of strongly continuous contractive $L^{1}$ semigroup for the heat equation was proved under the Ricci curvature assumption as in Theorem 2. However, the situation when there might be an isolated solution which does not come from a semigroup was not covered.

The sharpness of our curvature assumption in Theorem 2 can be seen by Azencott's [1] example. He constructed complete two-dimensional surfaces which have sectional curvature behaving like

$$
K(x) \sim-C r^{2+\varepsilon}(x)
$$

for $C, \varepsilon>0$, at infinity. Moreover these surfaces admit infinitely many fundamental solutions of the heat equation with the property that $K(x, y, t) \geqslant 0$ 
and

$$
\int_{M} K(x, y, t) d y \leqslant 1
$$

for all $x \in M, t \in[0, \infty)$. If we denote $H(x, y, t)$ to be the minimal fundamental solution obtained by taking the limit of the fundamental solutions with Dirichlet boundary condition of any compact exhaustion of $M$, then it is known that $H(x, y, t)<K(x, y, t)$ on $M \times M \times(0, \infty)$. Hence if we define

$$
f_{x}(y, t)=K(x, y, t)-H(x, y, t)
$$

for any fixed $x \in M$, then $f_{x}$ is a nonnegative $L^{1}$ solution of the heat equation with

$$
\lim _{t \rightarrow 0} \int_{M} f_{x}(y, t) d y=\lim _{t \rightarrow 0} \int_{M} K(x, y, t) d y-\lim _{t \rightarrow 0} \int_{M} H(x, y, t) d y=0 .
$$

However, clearly $f_{x} \not \equiv 0$. This gives a counterexample to the $L^{1}$ uniqueness of the heat equation.

Proof of Theorem 1. Let $g(x)$ be a nonnegative $L^{1}$ subharmonic function defined on $M$. We consider the solution of the heat equation

$$
\left(e^{\Delta t} g\right)(x)=\int_{M} H(x, y, t) g(y) d y
$$

with $g(x)$ as initial data. Partial differentiating with respect to $t$, we have

$$
\begin{aligned}
\frac{\partial}{\partial t}\left(e^{\Delta t} g\right)(x) & =\int_{M} \frac{\partial}{\partial t} H(x, y, t) g(y) d y \\
& =\int_{M}\left(\Delta_{y} H(x, y, t)\right) g(y) d y .
\end{aligned}
$$

We claim that integration by parts is valid and (5) becomes

$$
\frac{\partial}{\partial t}\left(e^{\Delta t} g\right)(x)=\int_{M} H(x, y, t) \Delta g(y) d y \geqslant 0 .
$$

To justify this, we argue by considering the integrals over the geodesic ball of radius $R$, centered at $x_{0}$. Green's identity yields

$$
\begin{aligned}
\left|\int_{B_{x_{0}}(R)}\left(\Delta_{y} H(x, y, t)\right) g(y) d y-\int_{B_{x_{0}}(R)} H(x, y, t) \Delta g(y) d y\right| \\
\quad=\left|\int_{\partial B_{x_{0}}(R)} \frac{\partial H}{\partial \nu_{y}}(x, y, t) g(y)-\int_{\partial B_{x_{0}}(R)} H(x, y, t) \frac{\partial g}{\partial \nu}(y) d y\right| \\
\leqslant \int_{\partial B_{x_{0}}(R)}\left|\nabla_{y} H\right|(x, y, t) g(y)+\int_{\partial B_{x_{0}}(R)} H(x, y, t)|\nabla g|(y) .
\end{aligned}
$$


Obviously, it suffices to show that both boundary integrals on the right-hand side vanish as $R \rightarrow \infty$. By considering only large values of $R$, we may assume that $x \in B_{x_{0}}(R / 4)$.

In [6, Theorem 2.1], it was shown that any nonnegative subharmonic function $g(x)$ must satisfy

$$
\sup _{B_{x_{0}}(R)} g(y) \leqslant C e^{\alpha \sqrt{k(R) R}} \operatorname{Vol}^{-1}\left(B_{x_{0}}(2 R)\right) \int_{B_{x_{0}}(2 R)} g(y) d y
$$

for some constants $C, \alpha>0$ depending only on $n$. The term $\operatorname{Vol}\left(B_{x_{0}}(2 R)\right)$ denotes the volume of the geodesic ball $B_{x_{0}}(2 R)$ and $-k(R)$ is the lower bound of the Ricci curvature on $B_{x_{0}}(10 R)$. Applying the curvature assumption, we have the estimate

$$
\sup _{B_{x_{0}}(R)} g(y) \leqslant C e^{\alpha R^{2}} \mathrm{Vol}^{-1}\left(B_{x_{0}}(2 R)\right)\|g\|_{L^{1}}
$$

Consider $\varphi(y)=\varphi(r(y))$ to be a cut-off function satisfying

$$
\varphi(r(y))= \begin{cases}1 & \text { on } B_{x_{0}}(R+1)-B_{x_{0}}(R), \\ 0 & \text { on } B_{x_{0}}(R-1) \cup\left(M-B_{x_{0}}(R+2)\right),\end{cases}
$$

with $0 \leqslant \varphi \leqslant 1$ and $|\nabla \varphi| \leqslant 3$. By the subharmonicity of $g$, we have

$$
\begin{aligned}
0 & \leqslant \int_{M} \varphi^{2}(y) g(y) \Delta g(y) d y=-2 \int_{M} \varphi g\langle\nabla \varphi, \nabla g\rangle-\int_{M} \varphi^{2}|\nabla g|^{2} \\
& \leqslant 2 \int_{M}|\nabla \varphi|^{2} g^{2}-\frac{1}{2} \int_{M} \varphi^{2}|\nabla g|^{2} .
\end{aligned}
$$

Therefore,

$$
\begin{aligned}
\int_{B_{x_{0}}(R+1)-B_{x_{0}}(R)}|\nabla g|^{2} & \leqslant 4 \int_{M}|\nabla \varphi|^{2} g^{2} \leqslant 12 \int_{B_{x_{0}}(R+2)} g^{2} \\
& \leqslant 12\|g\|_{L^{1}} \sup _{B_{x_{0}}(R+2)} g(y) \\
& \leqslant C e^{\alpha R^{2}} \operatorname{Vol}^{-1}\left(B_{x_{0}}(2 R+4)\right)\|g\|_{L^{1}}^{2} .
\end{aligned}
$$

On the other hand,

$$
\begin{aligned}
\int_{B_{x_{0}}(R+1)}-B_{x_{0}}(R) & |\nabla g| \\
& \leqslant\left(\int_{B_{x_{0}}(R+1)-B_{x_{0}}(R)}|\nabla g|^{2}\right)^{1 / 2} \operatorname{Vol}^{1 / 2}\left(B_{x_{0}}(R+1)-B_{x_{0}}(R)\right) \\
& \leqslant\left(\int_{B_{x_{0}}(R+1)-B_{x_{0}}(R)}|\nabla g|^{2}\right)^{1 / 2} \operatorname{Vol}^{1 / 2}\left(B_{x_{0}}(2 R+4)\right) .
\end{aligned}
$$


Hence, we deduce that

$$
\int_{B_{x_{0}}(R+1)-B_{x_{0}}(R)}|\nabla g| \leqslant C\|g\|_{L^{1}} e^{\alpha R^{2}}
$$

To control the heat kernel, we consider the estimate obtained in [7] by Yau and the author. It was proved [7, Theorem 3.3] that for any $\varepsilon>0$ there exists a constant $C(\varepsilon)>0$, such that

$$
\begin{aligned}
H(x, y, t) \leqslant & C(\varepsilon) \mathrm{Vol}^{-1 / 2}\left(B_{x}(\sqrt{t})\right) \mathrm{Vol}^{-1 / 2}\left(B_{y}(\sqrt{t})\right) \\
& \times \exp \left(\frac{-r^{2}(x, y)}{(4+\varepsilon) t}+\alpha \varepsilon\left(k(R)+R^{-2}\right) t\right)
\end{aligned}
$$

for $x, y \in B_{x_{0}}(R)$ and some constant $\alpha$ depending only on $n$. Applying a volume comparison argument (see [2]),

$$
\begin{aligned}
& \operatorname{Vol}\left(B_{x}(\sqrt{t})\right) \\
& \leqslant \operatorname{Vol}\left(B_{y}(r(x, y)+\sqrt{t})-B_{y}(r(x, y)-\sqrt{t})\right) \\
& \leqslant \operatorname{Vol}(B(\sqrt{t}))\left[\begin{array}{l}
\operatorname{Vol}(B(k(R), r(x, y)+\sqrt{t})) \\
-\operatorname{Vol}(B(k(R), r(x, y)-\sqrt{t}))
\end{array}\right] / \operatorname{Vol}(B(k(R), \sqrt{t})) \\
& \leqslant \operatorname{Vol}\left(B_{y}(\sqrt{t})\right) \frac{\operatorname{Vol}(B(k(R), r(x, y)+\sqrt{t}))}{\operatorname{Vol}(B(0, \sqrt{t}))} \\
& \leqslant \operatorname{Vol}\left(B_{y}(\sqrt{t})\right) \frac{C \exp (\sqrt{(n-1) k(R)}(r(x, y)+\sqrt{t}))}{t^{n / 2}}
\end{aligned}
$$

where $B(k(R), \sqrt{t})$ denotes the geodesic ball of radius $\sqrt{t}$ in the constant $-(n-1)^{-1} k(R)$ curvature, simply connected space form. Combining this with the assumption on the Ricci curvature, we deduce that

$$
\begin{aligned}
H(x, y, t) \leqslant & C \operatorname{Vol}^{-1}\left(B_{x}(\sqrt{t})\right) t^{-n / 4} \\
& \times \exp \left(\frac{-r^{2}(x, y)}{5 t}+\alpha\left(R^{2}+R^{-2}\right) t+\alpha R(r(x, y)+\sqrt{t})\right) .
\end{aligned}
$$


It was also proved in [3, Lemma 7 and (4.18)] that

$$
\begin{aligned}
& \int_{M-B_{x_{0}}(R)}|\nabla H|^{2}(x, y, t) d y \\
& \leqslant\left(\int_{M-B_{x_{0}}(R / 2)} H^{2}(x, y, t)\right)^{1 / 2} \frac{64}{R^{2}} H^{1 / 2}(x, x, 2 t) \\
&+C t^{-1} H^{1 / 2}(x, x, t) \\
& \leqslant C\left(R^{-2} \mathrm{Vol}^{-1 / 2}\left(B_{x}(\sqrt{2 t})\right)+t^{-1} \mathrm{Vol}^{-1 / 2}\left(B_{x}(\sqrt{t})\right)\right) \\
& \times \exp (k(R) t)\left(\int_{M-B_{x_{0}}(R / 2)} H^{2}(x, y, t)\right)^{1 / 2} .
\end{aligned}
$$

On the other hand, by Theorem 3.1 of [7],

$$
\begin{aligned}
\int_{M-B_{x_{0}}(R / 2)} H^{2}(x, y, t) & \leqslant C \operatorname{Vol}^{-1}\left(B_{x}(\sqrt{t})\right) \sup _{y \in M-B_{x_{0}}(R / 2)} \exp \left(\frac{-r^{2}(x, y)}{5 t}\right) \\
& =C \operatorname{Vol}^{-1}\left(B_{x}(\sqrt{t})\right) \exp \left(\frac{-\left(R-2 r\left(x_{0}, x\right)\right)^{2}}{20 t}\right)
\end{aligned}
$$

Hence together with (8) and (11), we have

$$
\begin{aligned}
& \int_{B_{x_{0}}(R+1)-B_{x_{0}}(R)}|\nabla H|(x, y, t) g(y) d y
\end{aligned}
$$

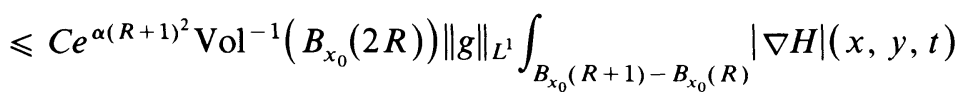

$$
\begin{aligned}
& \leqslant C e^{\alpha R^{2}}\|g\|_{L^{1}} \mathrm{Vol}^{-1}\left(B_{x_{0}}(2 R)\right) \mathrm{Vol}^{1 / 2}\left(B_{x_{0}}(R+1)-B_{x_{0}}(R)\right) \\
& \times\left(\int_{B_{x_{0}}(R+1)-B_{x_{0}}(R)}|\nabla H|^{2}(x, y, t)\right)^{1 / 2} \\
& \leqslant C\|g\|_{L^{1}} \mathrm{Vol}^{-1 / 2}\left(B_{x_{0}}(2 R)\right)\left(R^{-2}+t^{-1}\right) \mathrm{Vol}^{-1 / 2}\left(B_{x}(\sqrt{t})\right) \\
& \times \exp \left(\frac{-\left(R-2 r\left(x_{0}, x\right)\right)^{2}}{20 t}+R^{2} t\right) \text {. }
\end{aligned}
$$

By choosing $T$ sufficiently small, we deduce that for $t \in(0, T)$,

$$
\begin{aligned}
& \int_{B_{x_{0}}(R+1)-B_{x_{0}}(R)}|\nabla H|(x, y, t) g(y) d y \\
& \leqslant C(x, g) t^{-1 / 2} \mathrm{Vol}^{-1 / 2}\left(B_{x}(\sqrt{t})\right) \exp \left(-\beta R^{2}\right)
\end{aligned}
$$


for some fixed constant $\beta>0$ and $C(x, g)$ depending only on $n$, the point $x$ and the function $g$.

Similarly, we estimate the term

$$
\begin{aligned}
& \int_{B_{x_{0}}(R+1)-B_{x_{0}}(R)} H(x, y, t)|\nabla g|(y) d y \\
& \quad \leqslant\left(\sup _{y \in B_{x_{0}}(R+1)-B_{x_{0}}(R)} H(x, y, t)\right) \int_{B_{x_{0}}(R+1)-B_{x_{0}}(R)}|\nabla g|(y) d y \\
& \quad \leqslant C\|g\|_{L^{1}} e^{\alpha R^{2} \operatorname{Vol}^{-1}\left(B_{x}(\sqrt{t})\right) t^{-n / 4}} \\
& \quad \times \exp \left(\frac{-\left(R-r\left(x_{0}, x\right)\right)^{2}}{5 t}+\alpha\left(R^{2}+R^{-2}\right) t+\alpha R\left(R+r\left(x_{0}, x\right)+\sqrt{t}\right)\right),
\end{aligned}
$$

by applying (9) and (10). Also, by choosing $T$ sufficiently small, we have for $t \in(0, T)$

$$
\begin{aligned}
& \int_{B_{x_{0}}(R+1)-B_{x_{0}}(R)} H(x, y, t)|\nabla g|(y) d y \\
& \quad \leqslant C(x, g) t^{-n / 4} \mathrm{Vol}^{-1}\left(B_{x}(\sqrt{t})\right) \exp \left(-\beta R^{2}\right) .
\end{aligned}
$$

To prove the vanishing of the two boundary integrals of (7), we apply the mean value theorem to (12) and (13). Hence there exists $\bar{R} \in(R, R+1)$, such that,

$$
\begin{aligned}
\int_{\partial B_{x_{0}}(\bar{R})}|\nabla H|(x, y, t) g(y) d y+\int_{\partial B_{x_{0}}(\bar{R})} H(x, y, t)|\nabla g|(y) d y \\
\quad=\int_{B_{x_{0}}(R+1)-B_{x_{0}}(R)}(|\nabla H|(x, y, t) g(y)+H(x, y, t)|\nabla g|(y)) d y \\
\quad \leqslant C(x, g)\left[t^{-1 / 2} \mathrm{Vol}^{-1 / 2}\left(B_{x}(\sqrt{t})\right)+t^{-n / 4} \mathrm{Vol}^{-1}\left(B_{x}(\sqrt{t})\right)\right] \exp \left(-\beta R^{2}\right) .
\end{aligned}
$$

This establishes our claim, and (6) is valid for $t \in(0, T)$. However, the semigroup property gives

$$
\begin{aligned}
\frac{\partial}{\partial(s+t)}\left(e^{\Delta(s+t)} g\right) & =\frac{\partial}{\partial t}\left(e^{\Delta s} e^{\Delta t} g\right)=e^{\Delta s} \frac{\partial}{\partial t}\left(e^{\Delta t} g\right) \\
& =e^{\Delta s} e^{\Delta t}(\Delta g)=e^{\Delta(s+t)}(\Delta g),
\end{aligned}
$$

which implies that (6) is valid for all $t>0$.

For any $x \in M$, the function $e^{\Delta t} g(x)$ is a monotone increasing function in the $t$-variable because of (6). On the other hand, it was proved in [5] that with the assumption on the Ricci curvature, the kernel satisfies $\int_{M} H(x, y, t) d x=1$ 
for all $y \in M$ and $t>0$. Hence the $L^{1}$ norm of $e^{\Delta t} g$ can be computed as

$$
\begin{aligned}
\left\|e^{\Delta t} g\right\|_{L^{1}} & =\int_{M} \int_{M} H(x, y, t) g(y) d y d x \\
& =\int_{M} \int_{M} H(x, y, t) d x g(y) d y \\
& =\int_{M} g(y) d y=\|g\|_{L^{1}} \quad \text { for all } t>0 .
\end{aligned}
$$

This contradicts the fact that $e^{\Delta t} g$ is monotone in $t$, unless $e^{\Delta t} g=g$. In which case, the function $g$ must be harmonic to start out with.

To show $g$ is constant, we consider the function $g_{\gamma}=\min \{g, \gamma\}$ for any positive constant $\gamma$. By the harmoniticity of $g, g_{\gamma}$ is superharmonic. Also $0 \leqslant g_{\gamma} \leqslant g$ implies $g_{\gamma} \in L^{1}(M)$. Clearly, $g_{\gamma}$ satisfies the estimates which $g$ does, namely (8) and (9). Applying a similar argument which yields (6), we conclude that

$$
\frac{\partial}{\partial t}\left(e^{\Delta t} g_{\gamma}\right)=e^{\Delta t}\left(\Delta g_{\gamma}\right) \leqslant 0
$$

Hence $e^{\Delta t} g_{\gamma}$ now is a monotone decreasing sequence in $t$ and also $\left\|e^{\Delta t} g_{\gamma}\right\|_{L^{1}}=$ $\left\|g_{\gamma}\right\|_{L^{1}}$. This implies, again, $g_{\gamma}$ must be harmonic. By the regularity of harmonic functions, this is impossible unless $g_{\gamma} \equiv g$ or $g_{\gamma} \equiv \gamma$. Since $\gamma$ is an arbitrary nonnegative constant and $g$ is nonnegative, this implies $g$ must be identically constant.

To show the nonexistence of nonconstant $L^{1}$ harmonic functions we simply use the fact that the absolute value of a $L^{1}$ harmonic function is a nonnegative $L^{1}$ subharmonic function.

Proof of Theorem 2. Let $v(x, t)$ be a nonnegative function in $L^{1}(M)$ for all $t>0$, with

$$
\lim _{t \rightarrow 0} \int_{M} v(x, t) d x=0 \text { and }\left(\Delta-\frac{\partial}{\partial t}\right) v(x, t) \geqslant 0
$$

We consider the solution of the heat equation

$$
e^{\Delta t} v_{\varepsilon}(x)=\int_{M} H(x, y, t) v(y, \varepsilon) d y
$$

with $v(x, \varepsilon)$ as initial data. Define the function

$$
F_{\varepsilon}(x, t)=\max \left\{0, v(x, t+\varepsilon)-e^{\Delta t} v_{\varepsilon}(x)\right\} .
$$


This function is nonnegative and satisfies

$$
\lim _{t \rightarrow 0} F_{\varepsilon}(x, t)=0 \text { and }\left(\Delta-\frac{\partial}{\partial t}\right) F_{\varepsilon}(x, t) \geqslant 0 .
$$

By integrating the $t$-variable from 0 to $T$, we have $f(x)=\int_{0}^{T} F_{\varepsilon}(x, t) d t$, which satisfies

$$
\begin{aligned}
\Delta f(x) & =\int_{0}^{T} \Delta F_{\varepsilon}(x, t) d t \geqslant \int_{0}^{T} \frac{\partial F_{\varepsilon}}{\partial t}(x, t) d t \\
& =F_{\varepsilon}(x, T) \geqslant 0 .
\end{aligned}
$$

Moreover, its $L^{1}$ norm can be computed as

$$
\begin{aligned}
\int_{M} f(x) d x & =\int_{0}^{T} \int_{M} F_{\varepsilon}(x, t) d x d t \leqslant \int_{0}^{T} \int_{M}\left|v(x, t+\varepsilon)-e^{\Delta t} v_{\varepsilon}(x)\right| d x d t \\
& \leqslant \int_{0}^{T} \int_{M}|v(x, t+\varepsilon)| d x d t+\int_{0}^{T} \int_{M}\left|e^{\Delta t} v_{\varepsilon}(x)\right| d x d t<\infty .
\end{aligned}
$$

The first term on the right is finite by virtue of the assumption on $v$, and the second term is finite because $e^{\Delta t}$ is a contractive semigroup on $L^{1}(M)$. Applying Theorem 1 to the nonnegative $L^{1}$ subharmonic function $f(x)$, we conclude that $f$ is identically constant. However by $(15), F_{\varepsilon}(x, T)$ must be zero. Since $x$ and $T$ are arbitrary, the function $F_{\varepsilon}$ must be identically zero. This implies that

$$
e^{\Delta t} v_{\varepsilon}(x) \geqslant v(x, t+\varepsilon) .
$$

Now we consider the function

$$
e^{\Delta t} v_{\varepsilon}(x)=\int_{M} H(x, y, t) v(y, \varepsilon) .
$$

By applying the upper bound (10) of $H(x, y, t)$ and setting $R=1+2 r(x, y)$, we have

$$
\begin{aligned}
e^{\Delta t} v_{\varepsilon}(x) \leqslant & C t^{-n / 4} \mathrm{Vol}^{-1}\left(B_{x}(\sqrt{t})\right) \\
& \times \int_{M}\left[\exp \left(\frac{-r^{2}(x, y)}{5 t}+\alpha\left(r^{2}(x, y)+1\right) t\right) \times v(y, \varepsilon)\right] d y .
\end{aligned}
$$

For sufficiently small values of $t>0$, the right-hand side can be estimated by

$$
C t^{-n / 4} \operatorname{Vol}^{-1}\left(B_{x}(\sqrt{t})\right)\|v(\cdot, \varepsilon)\|_{L^{1}}
$$

Hence as $\varepsilon \rightarrow 0, e^{\Delta t} v_{\varepsilon}(x) \rightarrow 0$ since $\|v(\cdot, \varepsilon)\|_{L^{1}} \rightarrow 0$. However, by the semigroup property, $e^{\Delta t} v_{\varepsilon}(x) \rightarrow 0$ for all $x \in M$ and $t>0$. Together with (16), this shows $0 \geqslant v(x, t)$. By the assumption that $v$ is nonnegative, we conclude the vanishing of $v$. 
To prove that any $L^{1}$ solution is uniquely determined by its initial data, we simply consider its absolute value and apply the first half of the theorem.

We would like to remark that if $M$ is a simply connected manifold with nonpositive sectional curvature, then it was shown in [6] that $L^{1}$ uniqueness is valid for harmonic functions. The standard comparison theorem also implied that the heat kernel of $M$ is bounded above by the Euclidean heat kernel. Therefore, the argument to prove Theorem 2 can be carried over to establish $L^{1}$ uniqueness for the heat equation on simply connected nonpositive sectional curvature manifolds.

\section{References}

[1] R. Azencott, Behavior of diffusion semi-groups at infinity, Bull. Soc. Math. France 102 (1974) 193-240.

[2] J. Cheeger, M. Gromov \& M. Taylor, Finite propagation speed, kernel estimates for functions of the Laplace operator, and the geometry of complete Riemannian manifolds, J. Differential Geometry 17 (1983) 15-53.

[3] S. Y. Cheng, P. Li \& S. T. Yau, On the upper estimate of the heat kernel of a complete Riemannian manifold, Amer. J. Math. 103 (1981) 1021-1063.

[4] H. Donnelly, Uniqueness of positive solutions of the heat equation, preprint.

[5] L. Karp \& P. Li, The heat equation on complete Riemannian manifolds, preprint.

[6] P. Li \& R. Schoen, $L^{p}$ and mean value properties of subharmonic functions on Riemannian manifolds, Acta Math., to appear.

[7] P. Li \& S. T. Yau, On the parabolic kernel of the Schrödinger operator, preprint.

[8] R. Strichartz, Analysis of the Laplacian on a complete Riemannian manifold, J. Funct. Anal. 52 (1983) 48-79.

[9] S. T. Yau, Harmonic functions on complete Riemannian manifolds, Comm. Pure Appl. Math. 28 (1975) 201-228.

[10] Some function-theoretic properties of complete Riemannian manifolds and their applications to geometry, Indiana Univ. Math. J. 25 (1976) 659-670.

Purdue University 
